substrates also generated via a fatty acid (FA) biosynthetic cascade. Dietary studies using fish oil-derived EPA have been disappointing in UC; we hypothesised that the PUFA biosynthetic pathway in inflamed tissue is altered. This study evaluated PUFA profile in inflamed and non-inflamed mucosa from UC patients and compared to matched controls.

Methods Ethical approval was obtained. Patients were prospectively recruited from outpatients' clinics. Mucosal biopsies at flexible sigmoidoscopy (FS) were taken from UC patients within inflamed and normal proximal mucosa. Age-sex matched control patients undergoing FS for functional symptoms were compared. Inflammation was scored endoscopically and histologically. Membrane bound FA (MBFA): Biopsies were spiked with deuterated internal standard, followed by liquid-liquid extraction and quantitative gas chromatography mass spectrometry (MS). Free Fatty Acid (FFA): Biopsies were homogenised, followed by solid phase extraction and liquid chromatography orbitrap MS. Data were expressed as percentage abundance. Dietary fatty acid analysis was undertaken. Wilcoxon signed rank pair and Spearman's correlation analysis were employed. Results 69 active UC patients (54 paired normal/inflamed mucosa) and 69 controls were compared. No biologically significant differences were noted between endoscopically normal mucosa from UC patients and controls other than DPA $(p<0.0025)$. Inflamed mucosa compared to non-inflamed mucosa demonstrated highly significant reduction in LA and $\alpha$ LNA $(p<0.0001)$ and increased AA, DPA, and DHA $(p<0.0001)$; EPA was reduced $(p<0.005)$. The ratio of AA/EPA was increased in inflamed mucosa $(p<0.0001)$. These findings are consistent between MBFA and FFA and correlate with severity of inflammation.

Conclusion Mucosal PUFA bioavailability is altered in active UC, with significant elevation of AA and reduction of LA, aLNA and EPA. This suggests modification of the FA biosynthetic pathway with elevated delivery of AA as a precursor of pro-inflammatory eicosanoids in active UC. These findings may explain the lack of efficacy of supplemental fish oil and suggests new alternative therapeutic targets.

Competing interests None declared.

\section{PMO-249 OUANTITATIVE PROTEOMIC ANALYSIS OF INTERMEDIATE FILAMENT PROFILE IN ULCERATIVE COLITIS REVEALS INCREASED LEVELS OF KERATINS 8, 18 AND 19 IN PATIENTS WITH LONGSTANDING PAN COLITIS WHICH ARE REDUCED WITH DEVELOPMENT OF DYSPLASIA}

doi:10.1136/gutjnl-2012-302514b.249

\begin{abstract}
1,2D Majumdar,* ${ }^{2} \mathrm{~B} \mathrm{M}$ Corfe, ${ }^{3} \mathrm{C}$ Evans, ${ }^{1} \mathrm{~A} \mathrm{~J}$ Lobo. ${ }^{1}$ Gastroenterology, Royal Hallamshire Hospital, Sheffield, UK; ${ }^{2}$ Department of Oncology, University of Sheffield, Sheffield, UK; ${ }^{3}$ Department of Chemical and Biological Engineering, University of Sheffield, Sheffield, UK
\end{abstract}

Introduction Intermediate filaments (IF), principally keratins (K), are key components of epithelial cytoskeleton. K8, 18 and 19 are expressed in intestinal epithelial cells and play a role in cell-death signalling pathways, in particular apoptosis mediated by tumour necrosis factor- $\alpha$. Reduced K8 and K20 expression is linked to epithelial-to-mesenchymal transition indicative of increased tumour aggressiveness. We investigated the change in levels of insoluble IF proteins in well-characterised groups of patients at differing risk of UC-associated cancer.

Methods Rectal biopsies were obtained from patients with inactive UC with: (1) Long-standing (20-40 years) pancolitis (LSPC) $(n=10)$; (2) Recent onset ( $<5$ years) UC (ROUC) $(n=8)$; (3) UC with primary sclerosing cholangitis (PSC) $(n=7)$; (4) pancolitis with dysplasia $(n=4)$ and 10 controls, with additional biopsies from dysplastic/neoplastic lesions and snap frozen. An iTRAQ (isobaric tagging for relative and absolute quantification)-compatible extrac-

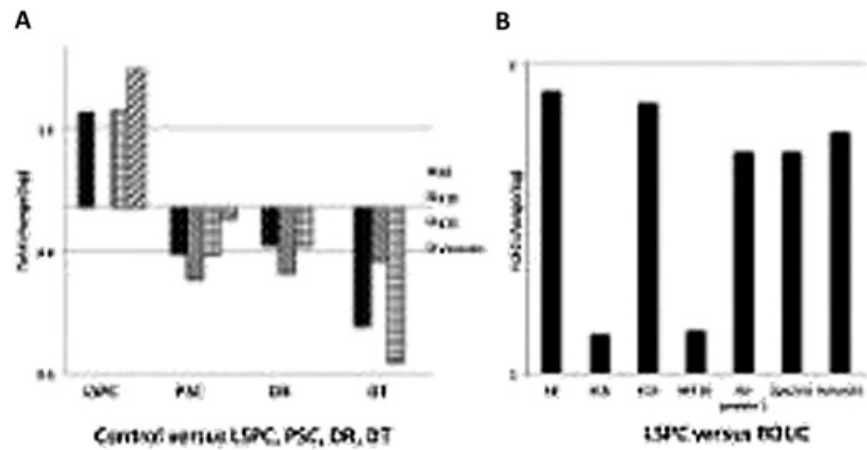

Abstract PM0-249 Figure 1 Tandem mass spectrometry results showing significant log fold changes $(p<0.05)$ in IF levels.

tion and solubilisation protocol for IF proteins was developed. Labelled peptides from pooled patients were analysed by SCX-LCMS/MS (strong cation exchange-reverse phase HPLC tandem mass spectrometry) and data reconstituted in GeneBio Phenyx. Intergroup comparisons were made using in-house algorithms based on t-testing with multiple test correction.

Results Tandem mass spectrometry (MS/MS) identified 52 proteins; $32(61.5 \%)$ were matched by two or more peptides. Abstract PMO249 figure $1 \mathrm{~A}$ shows the log fold change in IF levels compared to control, with significant increase in levels of K8, K19 and vimentin in those with LSPC, but marked reduction in IF levels in areas of dysplasia (DT) and rectal mucosa distant from this (DR). Marked increase in levels of keratins was noted in patients with LSPC compared to those with ROUC (Abstract PMO-249 figure 1B), suggesting an effect of disease duration on IF levels.

Conclusion This is the first study using a quantitative proteomic approach with an iTRAQ based proteomic workflow to analyse changes in IF levels in patients with UC with differing colon cancer risk. LSPC is associated with enhanced mucosal levels of keratins, spectrin and xin, which is reduced in dysplasia and in distant rectal mucosa of those with dysplasia-suggesting a field change. These changes need further characterisation including of posttranslational modifications, which may help better understanding of the pathogenesis of colitis associated cancer.

Competing interests None declared.

\section{PMO-250 QUANTITATIVE PROTEOMICS IN ULCERATIVE COLITIS REVEALS MUCOSAL INFLAMMATION REDUCES LEVELS OF KERATINS IN THE INSOLUBLE FRACTION OF THE INTERMEDIATE FILAMENT PROTEOME}

doi:10.1136/gutjnl-2012-302514b.250

${ }^{1,2} \mathrm{D}$ Majumdar, ${ }^{*}{ }^{3} \mathrm{C}$ Evans, ${ }^{2} \mathrm{~B}$ M Corfe, ${ }^{1} \mathrm{~A} \mathrm{~J}$ Lobo. ${ }^{1}$ Department of Gastroenterology, Royal Hallamshire Hospital, Sheffield, UK; ${ }^{2}$ Department of Oncology, University of Sheffield, Sheffield, UK; ${ }^{3}$ Department of Chemical and Biological Engineering, University of Sheffield, Sheffield, UK

Introduction Keratins (K) are a key component of intermediate filaments (IF), primarily composed of K8, 18 and 19 in the intestinal epithelia. Apart from a structural role, they may play a role in moderating TNF effects, including cytotoxicity. K8-null mice develop colitis, a subset of patients with IBD have missense mutation in $\mathrm{K} 8$ gene. Colonic expression of $\mathrm{K} 8 / \mathrm{K} 18$ has been shown to be regulated by IL-6. In order to examine the relationship between acute inflammation and alteration in levels of insoluble IFs in mucosa of patients with ulcerative colitis (UC), we undertook a quantitative proteomic approach using an iTRAQ (isobaric tagging for relative and absolute quantification)-based proteomic workflow.

Methods Endoscopic biopsies were obtained from patients with UC, from actively inflamed rectum and from non-inflamed proximal 
colonic mucosa in the same patient $(\mathrm{n}=10)$, and from normal controls $(n=10)$. Endoscopic (Baron) and histological assessment was made. An iTRAQ-compatible extraction protocol for insoluble IF proteins was developed. Labelled peptides from pooled patients were analysed by SCX-LC-MS/MS (strong cation exchange-reverse phase HPLC tandem mass spectrometry) and data reconstituted in GeneBio Phenyx. Inter-group comparisons were made using in-house algorithms based on t-testing with multiple test correction.

Results Median age was 36 years (range 23-71). Endoscopic Baron score was $\geq 2$ in inflamed mucosa for all patients. Tandem mass spectrometry (MS/MS) identified 52 proteins, 32 (61.5\%) matched by two or more peptides, showing significant log fold change, with reduced levels of keratins and vimentin in inflamed mucosa compared to controls (Abstract PMO-250 table 1). Abstract PMO250 figure 1 shows significantly reduced IF protein levels in inflamed mucosa compared to inactive mucosa. Cytokine proteins neutrophil defensin 1 and bone morphogenetic protein 4 were increased only in actively inflamed mucosa.

Abstract PM0-250 Table 1 Inflamed and non-inflamed mucosa vs controls (log fold change)

\begin{tabular}{llllll}
\hline Protein & Accession no & MolWeight (kDa) & Peptide no & Active & Inactive \\
\hline K8 & P05787 & 53.7 & 35 & $0.4^{*}$ & NS \\
K18 & P05783 & 48.0 & 17 & $0.7^{*}$ & NS \\
K19 & P08727 & 44.1 & 31 & $0.4^{*}$ & NS \\
Vimentin & B3KRK8 & 46.9 & 4 & $0.6^{*}$ & NS \\
\hline
\end{tabular}

${ }^{*} \mathrm{p}<0.05$.

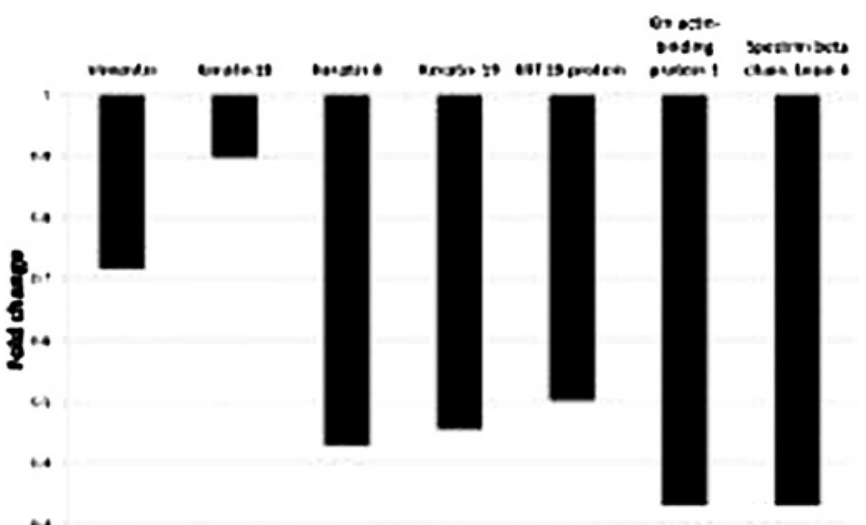

Abstract PM0-250 Figure 1 Decreased levels of IF proteins in inflamed colonic mucosa.

Conclusion Using a quantitative proteomic approach, we have shown significantly decreased levels of keratins in the actively inflamed colonic mucosa in UC providing further evidence of interaction between keratins and inflammatory pathways-which requires further elucidation.

Competing interests None declared.

\section{PM0-251 A RETROSPECTIVE AUDIT OF COLORECTAL CANCER SURVEILLANCE IN INFLAMMATORY BOWEL DISEASE IN SECONDARY CARE}

doi:10.1136/gutjnl-2012-302514b.251

K Burley, E Arthurs, ${ }^{*}$ B Gholkar, L Williams, M Lockett. Gastroenterology, North Bristol NHS Trust, Bristol, UK

Introduction Patients with colitis are at increased risk of colorectal cancer (CRC). Colonoscopic surveillance to detect dysplasia and early cancers has been advocated by the BSG since $2002{ }^{1}$ Our aims were to assess whether patients with colitis in our patient cohort are receiving appropriate colonoscopic surveillance for CRC according to these guidelines, and to assess the impact of the updated 2010 BSG guidelines ${ }^{2}$ on local endoscopy services.

Methods Patients with IBD were identified from secondary care coding databases and verified by paper records. A retrospective review of case notes was performed. Data on diagnoses, duration of symptoms, extent of disease and CRC surveillance was collected and analysed. Individualised recommendations for colonoscopic screening and surveillance were made according to the 2010 BSG guidelines.

Results 45 colitis patient records were reviewed; 20 CD: 25 UC, M:F 23:22. The average age was 59.4 (range 18.6-87); average duration of disease 18.6 (range $0-56) .35$ (78\%) had colitis extent requiring surveillance. 26 patients $(58 \%)$ had symptom onset $>10$ years; 11 patients (42\%) underwent screening colonoscopy at 8-10 years; 14 $(54 \%)$ did not, one patient underwent colonoscopy but date of diagnosis was unclear. Nine patients $(35 \%)$ underwent inconsistent surveillance, in six patients $(23 \%)$ there was no record of a colonoscopy. Reasons for inconsistent or absent surveillance included nonattendance (2), patient declined (1) and unclear (11). 24 patients were eligible for repeat colonoscopy; 3 (13\%) underwent this at the recommended interval; one patient was due in 2011; $11(46 \%)$ underwent inconsistent surveillance; nine (38\%) did not undergo any surveillance. Reasons for absent or inconsistent surveillance included non-attendance (2), lost to follow-up (1), patient declined (1), procedure unnecessary due to disease extent (1), patient undergoing surgery in the interval between colonoscopies (1) and unclear (14). Of 26 patients eligible for surveillance, 3 were excluded due to disease extent and intervening surgery. Of 23 remaining patients, the surveillance interval between colonoscopies would be increased in 12 patients (52\%), unchanged in $6(26 \%)$ and reduced in $3(13 \%)$ with the introduction of the 2010 BSG guidelines. The impact was unclear in two patients (9\%).

Conclusion Patients with colitis in our patient cohort at NBT are not receiving appropriate CRC surveillance according to BSG guidelines. These results emphasise the need for a robust coordinated surveillance programme. The 2010 guidelines have had the net effect of increasing the time interval between colonoscopies, which may lead to an overall reduction in endoscopy workload from surveillance cases.

Competing interests None declared.

\section{REFERENCES}

1. Eaden JA, Mayberry JF. Gut 2002;51(Suppl V):v10-12

2. Cairns SR, et al. Guidelines. Gut 2010;59:666-90.

\section{PMO-252 EFFICACY OF INFLIXIMAB THERAPY IN ACUTE AND SUB- ACUTE ULCERATIVE COLITIS}

doi:10.1136/gutjnl-2012-302514b.252

E Harrison,* J Slater, L Wood, S C Cooper, S de Silva. Department of Gastroenterology, Dudley Group of Hospitals NHS Foundation Trust, Birmingham, UK

Introduction Ulcerative colitis (UC) has a chronic relapsing course. Infliximab is beneficial in severe disease, but conflicting data exists regarding the subsequent colectomy rates. We aimed to review outcomes post-infliximab usage in acute and sub-acute UC in our clinical practice.

Methods We conducted a retrospective review of all patients who had received a maximum of three doses of infliximab for acute or sub-acute exacerbations of UC between January 2010 and October 2011. Medical treatment (initial and subsequent) and colectomy rates were recorded for all patients. 